Submitted to AJ, October 22, 2018

\title{
A strong jet/cloud interaction in the Seyfert galaxy IC 5063: VLBI observations
}

\author{
T.A. Oosterloo, R. Morganti ${ }^{1}$, \\ Netherlands Foundation for Research in Astronomy, Postbus 2, 7990 AA, Dwingeloo, NL \\ oosterloo@nfra.nl \\ morganti@nfra.nl \\ A. Tzioumis, J. Reynolds, E. King, \\ ATNF-CSIRO, P.O. Box 76, Epping NSW 1710, Australia \\ P. McCulloch, \\ Dept. of Physics, University of Tasmania, GPO Box 252-21, Hobart Tas 7001, Australia \\ and \\ Z. Tsvetanov \\ Johns Hopkins University, 3400 North Charles Street, Baltimore, MD 21218, USA
}

\begin{abstract}
We present 21-cm HI line and 13-cm continuum observations, obtained with the Australian Long Baseline Array, of the Seyfert 2 galaxy IC 5063. This object appears to be one of the best examples of Seyfert galaxies where shocks produced by the radio plasma jet influence both the radio as well as the near-infrared emission. The picture resulting from the new observations of IC 5063 confirms and completes the one derived from previous Australia Telescope Compact Array (ATCA) lower resolution observations. A strong interaction between the radio plasma ejected from the nucleus and a molecular cloud of the ISM is occurring at the position of the western hot spot, about $0.6 \mathrm{kpc}$ from the active nucleus. Because of this interaction, the gas is swept up forming, around the radio lobe, a cocoon-like structure where the gas is moving at high speed. Due to this, part of the molecular gas is dissociated and becomes neutral or even ionised if the UV continuum produced by the shocks is hard and powerful enough.
\end{abstract}

${ }^{1}$ Istituto di Radioastronomia, CNR, via Gobetti 101, 40129 Bologna, Italy 
In the 21-cm H I line new data, we detect only part of the strong blue-shifted H I absorption that was previously observed with the ATCA at lower resolution. In particular, the main component detected in the VLBI absorption profile corresponds to the most blue-shifted component in the ATCA data, with a central velocity of $2786 \mathrm{~km} \mathrm{~s}^{-1}$ and therefore blue-shifted $\sim 614 \mathrm{~km} \mathrm{~s}^{-1}$ with respect to the systemic velocity. Its peak optical depth is $5.4 \%$. The corresponding column density of the detected absorption, for a spin temperature of $100 \mathrm{~K}$, is $N_{\mathrm{HI}} \sim 2 \times 10^{21}$ atoms $\mathrm{cm}^{-2}$. Most of the remaining blue-shifted components detected in the ATCA HI absorption profile are now undetected, presumably because this absorption occurs against continuum emission that is resolved out in these high-resolution observations.

The H I absorption properties observed in IC 5063 appear different from those observed in other Seyfert galaxies, where the HI absorption detected is attributed to undisturbed foreground gas associated with the large-scale galaxy disk. In the case of IC 5063, only a small fraction of the absorption can perhaps be due to this. The reason for this can be that the western jet in IC 5063 passes through a particularly rich ISM. Alternatively, because of the relatively strong radio flux produced by this strong interaction, and the high spectral dynamic range of our observations, broad absorption lines of low optical depth as detected in IC 5063 may have remained undetected in other

Seyferts that are typically much weaker radio emitters or for which existing data is of poorer quality.

Subject headings: galaxies: individual(IC 5063) — galaxies: ISM — galaxies: Seyfert

\section{Introduction}

The study of the effects of interactions between the radio plasma ejected from an active nucleus and the interstellar medium (ISM) of the hosting galaxy is presently attracting a lot of interest. In particular, Seyfert and high-redshift radio galaxies appear to be the kind of objects where the effects of such interactions can be very important. They can range from shaping the morphology of the gas in the ISM (with the radio plasma sweeping up material as it advances in the ISM), to the ionisation of the gas itself. While there is little doubt on the presence of such interactions in objects like Seyferts or high- $z$ radio galaxies, the actual importance of these effects in determining the overall characteristics of these sources is still a matter of debate.

In some Seyfert galaxies the morphological association between the radio plasma and the optical line-emitting clouds, as well as the presence of disturbed kinematics in these clouds, is striking. In particular, the narrow-line regions (NLR) in Seyfert galaxies (i.e. regions of highly ionised, kinematically complicated gas emission that occupy the central area - up to $\sim 1 \mathrm{kpc}$ from the nucleus) often appears to form a 'cocoon' around the radio continuum emission (see e.g. Wilson 1997 for a review; Capetti et al. 1996; Falcke, Wilson \& Simpson (1998) and references therein). 
Moreover, outflow phenomena are observed in the warm gas of several Seyfert galaxies (see Aoki et al. 1996 for a summary). Thus, the NLRs represent some of the best examples of regions where interaction between the local ISM and the radio plasma takes place and can be studied in detail.

The situation appears to be different for the atomic hydrogen. Observations of the H I 21-cm line, in absorption, can trace the distribution of this gas in front of the brightest radio components, that are usually observed in the central region of Seyferts (of kpc or sub-kpc size, i.e. co-spatial with the NLRs). Thus, the study of the distribution and kinematics of the cold component of the circumnuclear ISM can nicely complement the optical data. Although H I absorption has been detected in a number of Seyfert galaxies (e.g. NGC 4151 Pedlar et al. 1992; NGC 5929, Cole et al. 1998; Mkn 6, Gallimore et al. 1998 ; see also Brinks \& Mundell 1996 and Gallimore et al. 1999 and references therein), most of the investigated objects show single localised H I absorption components that can be explained as rotating, inclined disks or rings aligned with the outer galaxy disk (Gallimore et al. 1999) and only very seldom with gas in a parsec-scale circumnuclear torus (NGC 4151, Mundell et al. 1995). These components are therefore originated by gas that is not in interaction with the radio plasma.

However, more complex H I absorption profiles that cannot be explained by the above mechanism have been observed in at least one Seyfert galaxy, IC 5063. Australia Telescope Compact Array (ATCA) observations of this galaxy (Morganti, Oosterloo \& Tsvetanov 1998, hereafter M98) have revealed a very interesting absorption system with velocities up to $\sim 700 \mathrm{~km} \mathrm{~s}^{-1}$ blue-shifted with respect to the systemic velocity. In this object, unlike in other Seyfert galaxies, at least some of the observed $\mathrm{H}$ I absorption is originating from regions of interaction between the radio plasma and the ISM, producing an outflow of the neutral gas. This object, therefore, poses a number of interesting questions as: where is the interaction occurring, what are the physical conditions, why such interaction is not seen more often in neutral gas in other Seyfert galaxies?

Previous H I observations were limited by low spatial resolution. In this paper we present the results from new VLBI observations aimed at investigating in more detail its nuclear radio structure and locating where the complex H I absorption observed with ATCA is really occurring.

Throughout the paper we adopt a Hubble constant of $H_{\circ}=50 \mathrm{~km} \mathrm{~s}^{-1}$, so that $1 \operatorname{arcsec}$ corresponds to $0.32 \mathrm{kpc}$ at the redshift of IC 5063.

\section{Summary of the properties of IC 5063}

IC 5063 is a nearby $(z=0.0110)$ early-type galaxy that hosts a Seyfert 2 nucleus that emits

particularly strong at radio wavelengths $\left(P_{1.4 \mathrm{GHz}}=6.3 \times 10^{23} \mathrm{~W} \mathrm{~Hz}^{-1}\right)$. This object has been recently studied, both in radio continuum at $8 \mathrm{GHz}$ and in the 21-cm line of $\mathrm{H} \mathrm{I}$, using the ATCA (M98). In the continuum, on the arcsecond scale, we find a linear triple structure (see Fig. 1) of about 4 arcsec size $(\sim 1.3 \mathrm{kpc})$, that shows a close spatial correlation with the optical ionised gas, very similar in nature to what is observed in several other Seyfert galaxies (see e.g. Wilson 1997) 
and indicating that the radio plasma is important in shaping the NLR.

In the H I 21-cm line, apart from detecting the emission from the large-scale disk of IC 5063, very broad $\left(\sim 700 \mathrm{~km} \mathrm{~s}^{-1}\right)$, mainly blue-shifted absorption was detected against the central continuum source. These line observations could only be obtained with $\sim 7 \operatorname{arcsec}$ resolution, the highest resolution achievable with the ATCA at this wavelength. This resolution is too low to resolve the linear continuum structure detected in the $8-\mathrm{GHz}$ continuum image. However, and what makes this absorption particularly interesting is that we were able to conclude (by a careful analysis of the data, see M98 for the detailed discussion) that at least the most blue-shifted absorption is likely to originate against the western (and brighter) radio knot and not against the central radio feature seen at $8 \mathrm{GHz}$. The large, blue-shifted velocities observed in the absorption profile make it very unlikely that these motions have a gravitational origin (the most blue-shifted H I emission associated with the large-scale H I disk occurs at roughly $300 \mathrm{~km} \mathrm{~s}^{-1}$ with respect to the systemic velocity), and are more likely to be connected to a fast outflow of the ISM caused by an interaction with the radio plasma.

The identification of the central radio feature as the core, and hence that the absorption is occurring against the western lobe, is an important element in interpreting the nature of the absorption detected in IC 5063. In the literature, the core of this galaxy has sometimes been identified with the bright western knot (Bransford et al. 1998), however in our opinion there is compelling evidence that the identification of M98 is correct.

The superposition of the $8-\mathrm{GHz}$ radio image with an optical WFPC2 image available from the HST public archive and with a ground based narrow-band [O III] image suggests that the nucleus coincides with the central radio knot (see Figs 3 and 4 from M98). Although, as usual, there is some freedom in aligning the WFPC2 image with the 8-GHz radio image, aligning the western radio knot with the nucleus would require too large a shift. Given that the WFPC2 image was taken through the F606W filter, it contains the bright emission lines of [O III] $\lambda 5007, \mathrm{H} \alpha$ and [N II] $\lambda \lambda 6548,6584$, and it gives a good idea of the morphology of the ionised gas. By aligning the nucleus with the central radio knot, a good overall correspondence between the radio morphology and the bright region of optical emission lines is obtained, both in the WFPC2 image and the ground-based [O III] image, similar in nature to what is observed in many other Seyfert galaxies. Choosing this alignment, the western radio knot falls right on top of a very bright, unresolved, spike in the WFPC2 image, i.e. the western radio knot would also have a counterpart in the WFPC2 image. The filamentary morphology of the ionised gas of the region just around this spike is suggestive of an interaction between the radio plasma and the ISM and the identification of the western radio lobe with this feature seems natural. Using optical spectroscopy, Wagner \& Appenzeller (1989) found off-centre blue-shifted broad emission lines with similar widths as the detected H I absorption at a position 1-2 arcsec west of the nucleus, i.e. coincident with the spike seen in the WFPC2 image. This also suggests that at this position a violent interaction is occurring.

The identification of the core with the central radio knot has been recently confirmed by 
Kulkarni et al. (1998) from NICMOS images. Three well resolved knots were detected in the emission lines of [Fe II], $\mathrm{Pa} \alpha$ and $\mathrm{H}_{2}$. This emission-line structure shows a direct correspondence with the radio continuum structure. In broad band near IR images they detected a very red point source coincident with the central source seen in the emission lines, consistent with previous suggestions of a dust-obscured active nucleus. The strong [FeII] and $\mathrm{H}_{2}$ emission are usually interpreted as evidence for fast shocks and the direct correspondence between these regions and the radio emission suggest that shocks associated with the radio jet play a role in the excitation of the emission-line knot.

By the same authors, an asymmetry in the $\mathrm{H}_{2}$ distribution was found, with the eastern lobe showing a much weaker emission than the western lobe. This asymmetry can be explained, e.g, if an excess of molecular gas is present on the western side (for example, if the radio jet has struck a molecular cloud).

In the optical, IC5063 shows a very high-excitation emission line spectrum (including [Fe VII] $\lambda \lambda 5721$, 6087; Colina, Spark \& Macchetto 1991). The high-excitation lines are detected within 1 - 1.5 arcsec on both sides of the nucleus, about the distance between the radio core and both the lobes. These lines indicate the presence of a powerful and hard ionising continuum in the general area of the nucleus and the radio knots in IC 5063. We have estimated (M98) the energy flux in the radio plasma to be an order of magnitude smaller than the energy flux emitted in emission lines. The shocks associated with the jet-ISM interaction are, therefore, unlikely to account for the overall ionisation and the NLR must be, at least partly, photoionised by the nucleus, unless the lobe plasma contains a significant thermal component (Bicknell et al. 1998).

\section{VLBI observations}

IC 5063 was observed with the Australian Long Baseline Array (LBA) initially in continuum at $13 \mathrm{~cm}(2.3 \mathrm{GHz})$, followed by spectral-line observations at the frequency corresponding to the redshifted $\mathrm{HI}$.

The 13-cm observations in June 1996 comprised five stations; Parkes (64 m), Mopra (22 m), the Australia Telescope Compact Array $(5 \times 22$-m dishes as tied-array), the Mount Pleasant 26$m$ antenna of the University of Tasmania and the Tidbinbilla 70-m antenna of the Canberra Deep Space Communications Complex (CDSCC) near Canberra. The observations used the S2 recording system to record a single $16 \mathrm{MHz}$ band in right-circular polarisation and were correlated at the LBA S2 VLBI correlator of the Australia Telescope National Facility at Marsfield, Sydney.

The 13-cm data were edited and calibrated using the AIPS processing system. After this, the data were exported to DIFMAP (Sheperd 1997) for model fitting and imaging. The final image is presented in Fig. 2 and was made with uniform weighting.

Although the observations were not phase-referenced, absolute position calibration for the 13- 
$\mathrm{cm}$ LBA image was extracted from the delay and rate data, allowing the radio image to be fixed at the $\sim 0.1$ arcsec level in each coordinate, adequate for registration with other images.

The 21-cm observations were made in September 1997 at the redshifted HI frequency of $1407 \mathrm{MHz}$, recording $16 \mathrm{MHz}$ bandwidths in each circular polarisation. The same array was used, except for the Tidbinbilla 70-m antenna, which has no $21 \mathrm{~cm}$ capability. Correlation was in spectral-line mode with 256 spectral channels on each baseline and polarisation.

The editing and part of the calibration of the 21-cm line data was done in AIPS and then the data were transfered to MIRIAD (Sault, Teuben \& Wright 1995) for the bandpass calibration. The calibration of the bandpass was done using additional observations of the strong calibrators PKS 1921-293 and PKS 0537-441.

Problems were encountered at Mopra which limited the usefulness of those data. It proved not possible to image the source from the final dataset and instead a simpler analysis using the time-averaged baseline spectra was employed.

\section{The sub-kpc structure}

\subsection{The radio continuum morphology}

The final 13-cm image, shown in Fig. 2, has a beam of $\sim 56 \times 15$ mas in position angle (p.a.) $-40^{\circ}$. The r.m.s. noise is $\sim 0.7 \mathrm{mJy}_{\text {beam }}{ }^{-1}$. The total flux is $210 \mathrm{mJy}$. Because of the high accuracy of the astrometry of this VLBI image, we know that the observed structure corresponds (as expected) to the brighter, western, lobe observed in the 8-GHz ATCA image (see Fig. 2). It is therefore situated at about $0.6 \mathrm{kpc}$ from the nucleus.

The image shows that the lobe appears to have a relatively bright peak $\left(77 \mathrm{mJy}_{\mathrm{beam}}{ }^{-1}\right)$ and some extended emission to the north-east in p.a. $\sim 40^{\circ}$ of total size of about 50 mas (or $\sim 16 \mathrm{pc}$ ). The p.a. is quite different from the p.a. of the arcsecond sized structure seen in the ATCA 8-GHz data (p.a. $\sim 295^{\circ}$ ), so there appears to be structure perpendicular to the main radio structure. These kind of distortions are often seen in the radio structure of Seyfert galaxies (e.g. Falcke et al. 1998) and could perhaps result from the interaction of the radio plasma with the environment.

From our data, a brightness temperature of $\mathrm{T}_{\mathrm{B}} \sim 10^{7} \mathrm{~K}$ can be inferred for the VLBI source. This brightness temperature is several orders of magnitude less than the typical values seen in milliarcsecond AGN cores or inner (pc-scale) jets that typically have brightness temperatures between $10^{9}$ and $10^{11} \mathrm{~K}$. However, this temperature is quite commonly found for radio knots detected in Seyfert galaxies (e.g. knot C in NGC 1068, Roy et al. 1998). Unfortunately, we do not have a spectral index of this region on the VLBI scale. The overall spectral index inferred from the ATCA 8.6 and 1.4-GHz images is steep, $\alpha \sim-1$, and indeed consistent with a radio lobe or jet. However, unless a detailed multi-frequency spectral index study can be carried out, it is difficult to derive 
conclusions from this result alone given the complexity often observed in the spectral index of the central regions of Seyfert galaxies.

In summary, we can conclude that the radio morphology, the spectrum and the brightness temperature of the VLBI source are consistent with what expected in a radio lobe.

\subsection{The $\mathrm{H}$ I absorption}

As mentioned above, because from the 21-cm line observations useful data could only be obtained on the Parkes-ATCA baseline, we will present only an time-integrated spectral profile of the $\mathrm{H}$ I on this baseline. These data correspond to a spatial scale of about 0.1 arcsec.

Fig. 3 shows the continuum-weighted H I absorption profile. Heliocentric, optical velocities are used. For comparison, the spectrum obtained from the previous ATCA observations (with much lower spatial resolution) is superimposed (dashed line). In Fig. 3 we have also indicated the range of the velocities observed as measured for the HI emission of the large-scale disk of IC 5063, as well as the systemic velocity of the galaxy of $3400 \mathrm{~km} \mathrm{~s}^{-1}$ as derived from the kinematics of the H I emission. The r.m.s. limit to the optical depth is $\sim 0.3 \%$.

Fig. 3 shows that a strong absorption signal is detected against the VLBI source. Since from the 13-cm data it followed that the VLBI source corresponds to the western radio lobe, these data now confirm what was believed to be the case from the ATCA data, namely that the absorption is occurring against the western radio lobe. Fig. 4 shows the same data as in Fig. 3, except that both profiles have been normalised to the same optical depth for the most blue-shifted component.

Figs 3 and 4 show quite clearly that the shape of the absorption profile obtained at the high resolution of the VLBI data is quite different in character than that obtained with the ATCA. While in the ATCA data the absorption is relatively uniform in velocity, in the VLBI spectrum the most blue-shifted component is clearly the dominant one. This shows that the most blue-shifted absorption is occurring against a compact radio source, while the absorption at lower velocities is against a more diffuse source. Component $(A)$ has a central velocity of $2786 \mathrm{~km} \mathrm{~s}^{-1}$, over $600 \mathrm{~km}$ $\mathrm{s}^{-1}$ blue-shifted with respect to the systemic velocity $\left(3400 \mathrm{~km} \mathrm{~s}^{-1}\right)$, with its bluest wing extending

to about $2650 \mathrm{~km} \mathrm{~s}^{-1}$, or $-750 \mathrm{~km} \mathrm{~s}^{-1}$ relative to the systemic velocity. Component $A$ corresponds to the most blue-shifted component found in the ATCA profile, as is illustrated in Fig. 4. At slightly less blue-shifted velocities, but still outside the range of velocities observed in emission, the VLBI data show a second component $(B)$. The absorption with velocities within the range of the HI emission, as detected in the ATCA profile, is only partly detected in the VLBI spectrum with component $C$. No absorption is detect in the velocity range $3000-3200 \mathrm{~km} \mathrm{~s}^{-1}$. Hence, the absorption seen in the ATCA data at velocities above $3000 \mathrm{~km} \mathrm{~s}^{-1}$ has become much less prominent compared to the more blue-shifted absorption. Note that this effect is probably even stronger than the data shows, since the low resolution of the ATCA will have caused some filling of the absorption with emission of the H I disk and the 'true' absorption is likely to be stronger at these velocities. 
The ATCA spectrum also showed a faint red-shifted absorption component that is perhaps also detected in the VLBI spectrum.

The column density $N_{\mathrm{HI}}$ of the obscuring neutral hydrogen is given by $N_{\mathrm{HI}}=1.823 \times 10^{18} T_{s} \int \tau d v$ $\mathrm{cm}^{-2}$ where $T_{\mathrm{S}}$ is the spin temperature of the electron. Assuming a spin temperature of $100 \mathrm{~K}$ we derive a column density of $\sim 1.7 \times 10^{21}$ atoms $\mathrm{cm}^{-2}$ for the components $A$ and $B$ and a column density of $\sim 2.5 \times 10^{20}$ atoms $\mathrm{cm}^{-2}$ for the component $C$. The main source of uncertainty for the derived column density comes from the assumption in the value of the spin temperature. The presence of a strong continuum source near the $\mathrm{H}$ I gas can make the radiative excitation of the H I hyperfine state to dominate over the, usually more important, collisional excitation (see e.g. Bahcall \& Ekers 1969). Gallimore et al. (1999) argue that the H I causing the absorption against Seyferts jets is in general at too high densities $\left(\sim 10^{5} \mathrm{~cm}^{-3}\right)$ for these effects to be relevant. However, the argument used by Gallimore et al. applies to H I in pressure equilibrium with the NLR, while the absorbing gas in Seyferts in general is not co-spatial with the NLR, but is at larger radii. Because of this the density of the absorbing gas is lower, but the regions are also further removed from the central engine and the spin temperature approaches the kinetic temperature at much lower densities. In our model for IC 5063, the H I causing the most blue-shifted absorption is the skin of a molecular cloud that is being stripped off by the jet (see also §5). Given that typical densities in molecular clouds are in the range $10^{4}-10^{6} \mathrm{~cm}^{-3}$, this is an upper limit to the density of the absorbing gas. But given the large velocities involved, the actual density of the blue-shifted gas could be substantially lower.

The effects on the excitation of the fine-structure line by the local radiation field were already discussed by M98 for the case of IC 5063, where it was concluded that these effects are perhaps important. The column density derived by Kulkarni et al. from the NICMOS observation $\left(N_{\mathrm{HI}} \sim\right.$

$5 \times 10^{21}$ atoms $\mathrm{cm}^{-2}$ ) is slightly higher than our estimate based on a $T_{\text {spin }}$ of $100 \mathrm{~K}$, also suggesting that perhaps the spin temperature is somewhat higher than $100 \mathrm{~K}$. For $T_{\text {spin }}=100 \mathrm{~K}$ the derived column density is much lower than the value of $\sim 10^{23}$ atoms $\mathrm{cm}^{-2}$ found from X-ray data (Koyama et al. 1992).

\section{H I, $\mathrm{H}_{2}$ and radio plasma: a possible scenario for the interaction}

Summarising, the main result from our new observations is that with the improved spatial resolution, the absorption at velocities outside the range allowed by the rotational kinematics of the large-scale H I disk has become much stronger, while the absorption in the range of velocities of the H I disk has become much less prominent.

From the 13-cm radio continuum VLBI data we have been able to image only the western part of the source observed by the ATCA, while the remaining structure is resolved out.

Thus, all this confirms and completes the picture we derived from the ATCA data, namely that a strong interaction between the radio plasma and the ISM is occurring at the position of the 
western radio lobe.

Fig. 5 gives a schematic diagram of what we believe is happening in the western lobe of IC 5063. Following the results from NICMOS (Kulkarni et al. 1998), it is likely that the asymmetry observed in the brightness of the $\mathrm{H}_{2}$ (western side brighter than the eastern side) may be explained by an excess of molecular gas on the western side. Thus, the radio plasma ejected from the nucleus appears to interact directly with such a molecular cloud. Because of this interaction, the jet is drilling a hole in the dense ISM, sweeping up the gas and forming a cocoon-like structure around the radio lobe where the gas is moving at high speed and an outflow of gas is created. The increased ultraviolet radiation due to the presence of shocks generated from the interaction, can dissociate part of the molecular gas. This creates neutral hydrogen or even ionised gas if the UV continuum produced by the shocks is hard and powerful enough. The region of ionised gas would correspond to the part of the cocoon closer to where the interaction is occurring, possibly corresponding to both the shocked gas and to the precursor. The complex kinematics of the emission lines in this region observed from the optical emission lines (Wagner \& Appenzeller 1989) is consistent with this.

As for the neutral gas, we will observe only the component in front of the radio continuum and therefore, as effect of the outflow produced by the interaction, we will observe only the blueshifted component. The most blue-shifted component will be seen against the hot spot where the interaction is most intense. Somewhat away from this location, the H I will driven out by the expanding cocoon, but since this is away from the hot spot, this will occur at lower velocities. Moreover, the radio continuum emission from this region is also more extended compared to the small-sized hot spot. Hence the VLBI observation do not detect the absorption at lower velocities, but only the highest velocities against the hot spot (as illustrated in Fig.5).

The origin of the $\mathrm{H}_{2}$ emission can be related to UV or shocks (Draine, Roberge \& Dalgano 1983, Sternberg \& Dalgano 1989). Although we are not able to distinguish between these two mechanisms, this scenario suggest that there should be in IC 5063 a strong shock component. The $\mathrm{H}_{2}$ emission observed by NICMOS would therefore come from the very dense region (again due to the compression of the gas associated with the interaction) of the molecular cloud.

As we noticed above, not all the components observed in the ATCA data are also visible in the VLBI data. A possible explanation for this is that the components that are missing from the VLBI H I absorption are against continuum emission that is resolved out in our VLBI data, indicating that the cocoon of shocked gas is quite extended and cover at least all the western radio lobe. Alternatively, part of the absorption undetected in the VLBI spectrum can be due to the largescale disk associated with the dust-lane and also seen in H I in emission, although the continuity of the ATCA absorption profile does not suggest this.

By looking at the velocity field derived for this disk from the $\mathrm{H}$ I emission observations (see Fig. 5 in M98) we can see as the western side is the approaching side, therefore showing a blue-shifted velocity relative to the systemic. This means that the large-scale disk being in front of the hot spot 
could be responsible for component $C$, but that it cannot explain the weak red-shifted component unless non circular motions are present in the foreground gas associated with the dust lane. In M98 we hypothesised that the red-shifted component could be associated with a nuclear torus/disk. This was motivated by the fact that the width of the red-shifted component appeared to be similar to the width of the CO profile as observed by Wiklind et al. (1995). However, there seems to be no indication of detection of the nuclear component from the visibility of the continuum associated with the 21-cm data (and by extrapolating the arcsec data, the core flux is probably too weak to be detected and, even more, to produce an absorption) so this hypothesis has to be ruled out. A final possibility is that, apart from the bulk outflow, turbulent motions produced in the shocked region can give rise to clouds with red-shifted velocity.

\section{Comparison with other Seyfert galaxies}

How does IC 5063 compare with other Seyferts galaxies? The results on IC 5063 confirm the more general results obtained by Gallimore et al. (1999) on a sample of Seyfert galaxies, that the $\mathrm{HI}$ absorption is not occurring against the core and that the absorbing gas in Seyferts does not trace (except for NGC 4151) the pc-scale gas. In the galaxies studied by Gallimore et al., the absorption is occurring at a few hundred parsec from the core and is caused by the inner regions of, or gas associated with, the large-scale H I disks. This is also happening in IC 5063. The important difference is that in IC 5063 the jet is physically strongly interacting with this H I disk, causing the fast outflow observed for the absorbing material. This makes IC 5063 unique. Only component $C$ would exactly fit the scenario proposed by Gallimore et al. It is quite likely a gas cloud at large radius (given its column density), unrelated to the interaction, projected in front of the radio hot spot.

One obvious question is why such kind of absorption (i.e. broad blue-shifted absorption) is so rare. Are the physical conditions in IC 5063 rare, or is there an observational bias?

Some arguments suggest that IC 5063 is a special case. IC 5063 is a very strong radio emitter compared to other Seyfert galaxies. Most of the strong radio flux of IC 5063 is produced in the western radio knot, indicating that the interaction is particularly strong. Also the fact that the western radio knot is much brighter than the eastern one indicates that the conditions near the western lobe are special. It has been noted that IC 5063 belongs to a group of "radio-excess infrared galaxies" (Roy \& Norris 1997), objects that could represent active galactic nuclei hosted in an unusual environment or perhaps dust-enshrouded quasars or their progenitors. It appears that the jet-cloud interaction in IC 5063 is particularly strong. This would make IC 5063 a very suitable object for further detailed studies of jet-cloud interactions in Seyfert galaxies.

One factor is of course that in order to create the strong interaction and the very broad absorption, the jet has to lie more or less in the plane of the H I disk. Only then can the jet have a strong interaction with the ISM. The orientation of the AGN in Seyferts is not correlated with 
that of the large-scale disk, so the effects seen in IC 5063 should then only occur in a minority of cases.

On the other hand, interactions between the radio plasma and the ISM are common in Seyferts, given that in many Seyfert galaxies, very large velocity widths of the optical emission lines are observed in the NLR (e.g. Aoki et al. 1996 and references therein). Perhaps the high sensitivity in $\tau$ of our observations also plays a role. IC 5063 is a strong radio source compared to other Seyfert galaxies that are between 10 and 100 times weaker at radio wavelengths. Therefore only H I absorption with much higher optical depth can be observed against those objects. For example, the Seyfert 2 galaxy NGC 5929 shows a striking morphological similarity (both in the optical and radio) with IC 5063. However, the peak of the radio emission in NGC 5929 is only $24 \mathrm{mJy}_{\text {beam }}^{-1}$, so in this object absorption of a few percent would not be detectable with the noise level of the current observations (Cole et al. 1998). For almost all the galaxies in the sample studied by Gallimore et al. (1999) is the sensitivity not enough to have been able to detect faint, broad absorption like in IC 5063. Moreover, in order to detect broad profiles of the level as in IC 5063 even in strong sources, good spectral dynamic range is required, which is not always easy to obtain (e.g. NGC 1068; Gallimore et al. 1999). It is quite well possible that more cases like IC 5063 will be found if more sensitive observations are performed.

\section{Conclusions}

Using the Australian Long Baseline Array, we have detected a compact radio source of about of 50 mas (or $\sim 16 \mathrm{pc}$ ) in size (at $13 \mathrm{~cm}$ ) in the Seyfert galaxy IC 5063. Because of the high positional accuracy of these measurements, we can unambiguously identify this radio knot with the western radio lobe. The hot spot is extended in a direction almost perpendicular to the radio jet.

In 21-cm line observations, we detect absorption very much blue-shifted $\left(\sim 700 \mathrm{~km} \mathrm{~s}^{-1}\right)$ with respect to the systemic velocity. Together with the $13-\mathrm{cm}$ observations, this confirms that the H I absorption is not taking place against the core, but that it is against the western radio knot. At the position of the western radio knot a very strong interaction must be occurring between the radio jet and the ISM. Various arguments suggest that this interaction is particularly strong compared to other Seyfert galaxies. This makes IC 5063 a good candidate for studying the physics of jet-cloud interactions in Seyfert galaxies.

The HI absorption characteristics of IC 5063 are only partially consistent with other absorption

studies of Seyfert galaxies. The major absorption component is occuring against the bright radio knot offset a few hundred parsecs from the core. While there are indication that the absorbing material is associated with the large scale HI disk, it is clearly (and violently) disturbed by the passage of the jet. We suspect that more sensitive observations may reveal similar absorption profiles in other Seyfert galaxies with fainter radio sources. 
We wish to thank the referee, Jack Gallimore, for his useful comments. 


\section{REFERENCES}

Aoki K., Ohtani H., Yoshida M. \& Kosugi G. 1996, AJ 111, 140

Bahcall J.N., Ekers R.D. 1969, ApJ 157, 1055

Bicknell G.V., Dopita M. A., Tsvetanov Z.I., Sutherland R. S. 1998, ApJ 495, 680

Bransford M.A., Appleton P.N., Heisler C.A., Norris R.P., Marston A.P. 1998, ApJ 497, 133

Brinks, E., \& Mundell, C. G. 1996, in The Minnesota Lectures on Extragalactic Neutral Hydrogen, ed. E. D. Skillman, ASP Conf. Series, 106, 268

Capetti A., Macchetto F., Axon D.J., Sparks W.B. \& Boksenberg A. 1996, ApJ 469, 554

Cole G.H.J., Pedlar A., Mundell C.G., Gallimore J.F. \& Holloway 1998, MNRAS 301,782

Colina L., Sparks W.B. \& Macchetto F. 1991, ApJ 370, 102

Draine B.T., Roberge W.G. \& Dalgano A. 1983, ApJ 264, 485

Falcke H., Wilson A.S., Simpson C. 1998, ApJ 502, 199

Gallimore J.F., Holloway A.J., Pedlar A., Mundell C.G. 1998, A\&A 333, 13

Gallimore J.F., Baum S.A., O‘Dea C.P., Pedlar A., Brinks E. 1999, ApJ in press (astro-ph/9905267)

Koyama, K., Awaki, H., Iwasawa, K., \& Ward, M. J. 1992, ApJ, 399, L129

Kulkarni V.P. et al. 1998, ApJ 492, L121

Morganti R., Oosterloo T. \& Tsvetanov Z., 1998, AJ, 115, 915 (M98)

Pedlar A., Howley P., Axon D.J. \& Unger S.W., 1992, MNRAS, 259, 369

Mundel C.G., Pedlar A., Baum S.A., O’Dea C.P., Gallimore J.F. \& Brinks E. 1995, MNRAS 272, 355

Roy A.L., Colbert E.J.M., Wilson A.S., Ulvestad J.S. 1998, ApJ 504, 147

Roy A.L. \& Norris R.P. 1997, MNRAS 289, 824

Sault R.J., Teuben P.J., Wright M.C.H. 1995, in "Astronomical Data Analysis Software and Systems $I V$ ”, eds. R. Shaw, H.E. Payne and J.J.E. haynes, ASP Conf. Series, 77, 433

Shepherd M.C. 1997, in "Astronomical Data Analysis Software and Systems IV", ASP Conf. Series Vol. 125, Hunt G. \& Payne H.E. (eds.), p.77

Sternberg A. \& Dalgano A. 1989, ApJ 338, 197 
Wagner S.J. \& Appenzeller I., 1989, A\&A, 225, L13

Wiklind T., Combes F. \& Henkel C. 1995, A\&A 297, 643

Wilson A.S. 1997, in "Emission lines in Active Galaxies: new methods and techniques", eds. B.M. Peterson, F.-Z. Cheng and A.S. Wilson, ASP Conf. Series Vol. 113, p. 264

This preprint was prepared with the AAS $\mathrm{LAT}_{\mathrm{E}} \mathrm{X}$ macros v5.0. 
Fig. 1.- ATCA $3 \mathrm{~cm}$ radio continuum image from Morganti et al. (1998).

Fig. 2.- VLBI $13 \mathrm{~cm}$ radio continuum image of (the western lobe of) IC 5063

Fig. 3.- VLBI H I absorption profile converted in optical depth. For comparison, the ATCA profile is superimposed as dashed line.

Fig. 4.- VLBI (solid line) and ATCA (dashed line) H I absorption profile normalised to the same optical depth of the main absorption component.

Fig. 5. - Schematic diagram of the model presented in $\S 5$ to explain the characteristics of the H I absorption observed in the western lobe of IC 5063. See text for details. 


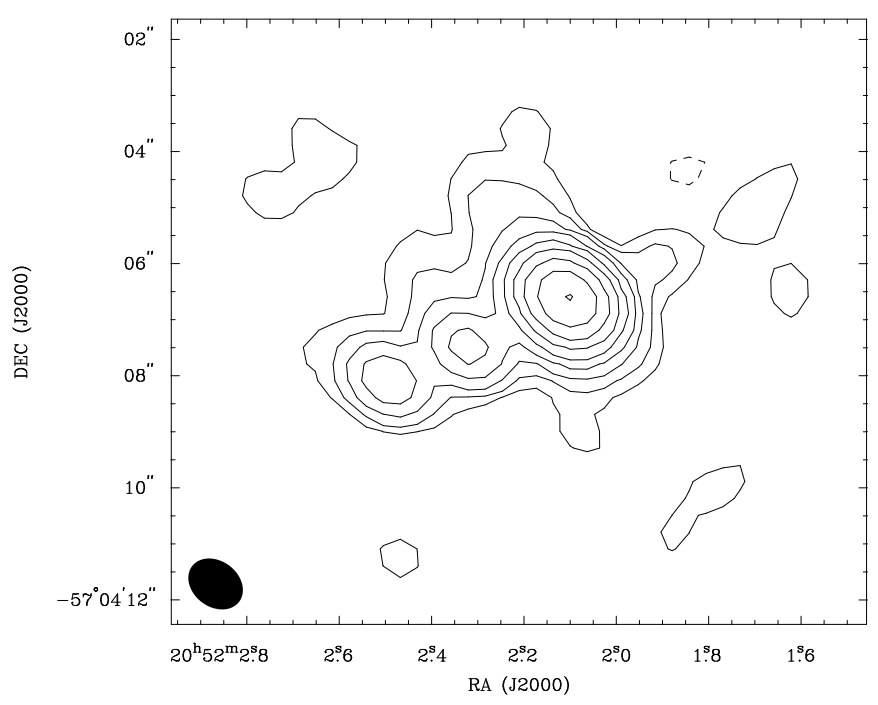

Fig.1

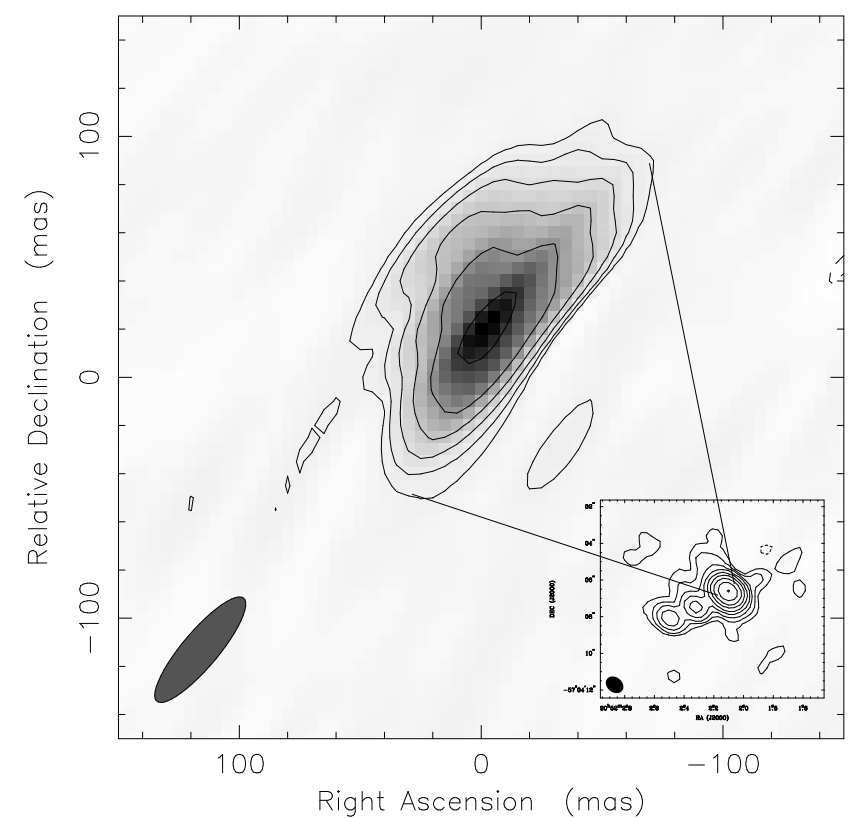

Map center: RA: 2052 02.102, Dec: -570406.602 (2000.0)

Map peak: $0.0767 \mathrm{Jy} /$ beam

Contours \%: -2.5 2.5 510204080

Beam FWHM: $55.9 \times 14.9$ (mas) at $-40^{\circ}$

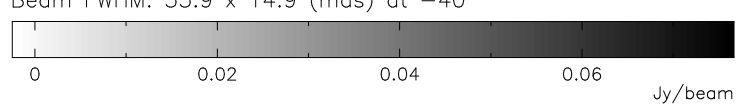

Fig.2 


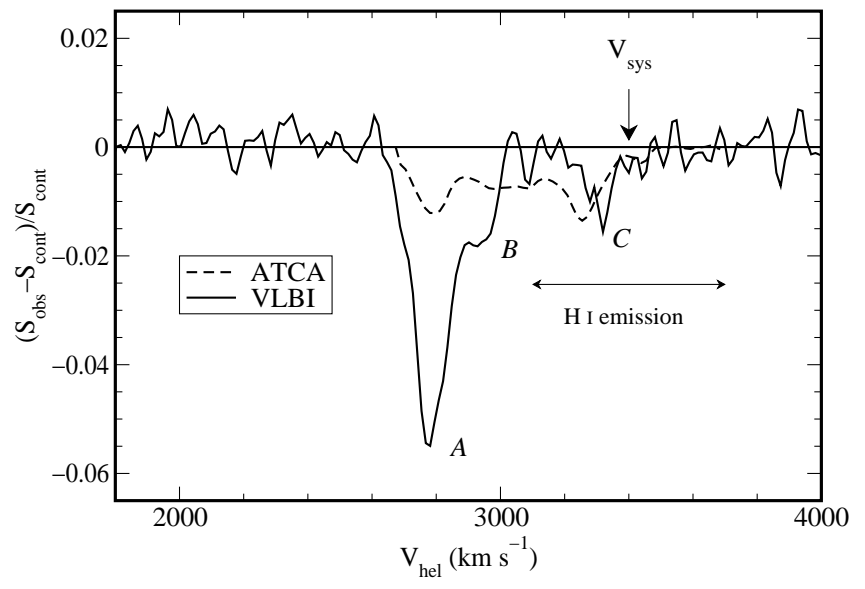

Fig.3

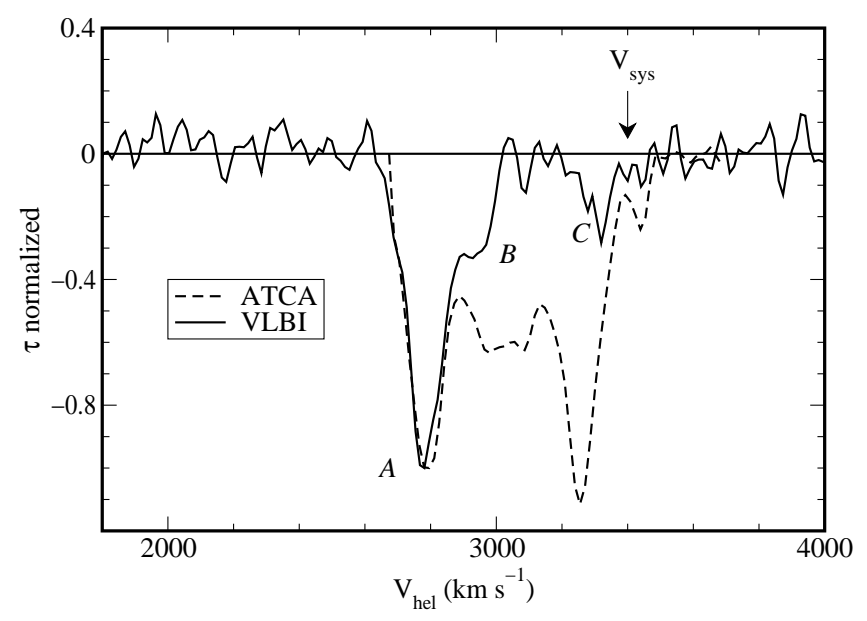

Fig.4 


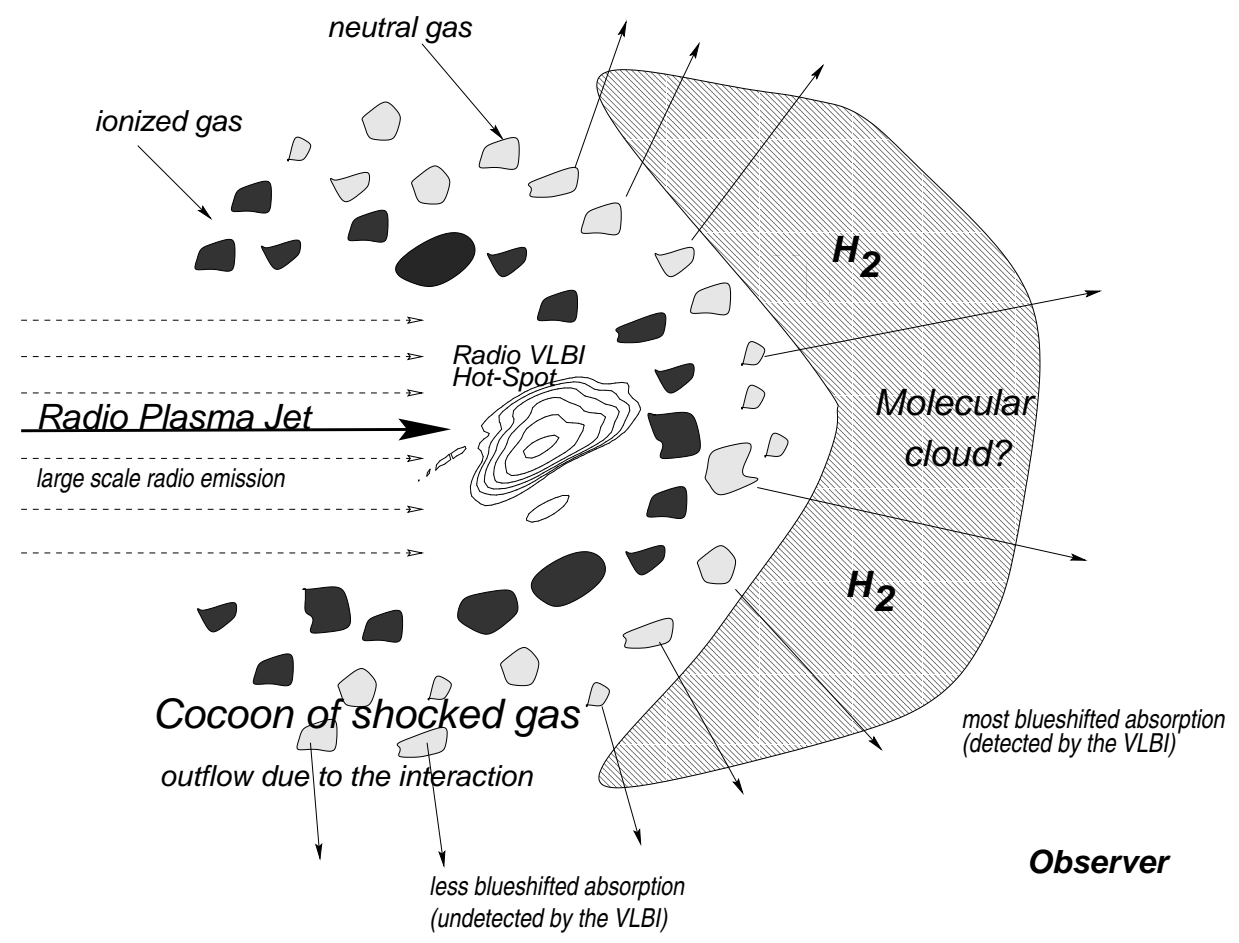

Fig.5 